\title{
Primary Nasopharyngeal Neuroendocrine Carcinoma: An Extremely Rare Clinical Entity
}

\author{
Yetkin Zeki YILMAZ1 ${ }^{1}$, Haluk ÇEVİK${ }^{1}$, Firat TEVETOĞLU ${ }^{1 *}$, \\ Cansu YOL ${ }^{2}$, Ayşegül BATIOĞLU KARAALTIN ${ }^{1}$
}

\begin{abstract}
Head and neck neuroendocrine carcinomas are rare, and the most common site of origin in this region is the larynx. Primary nasopharyngeal neuroendocrine carcinoma is very rare, and the number of cases reported so far is 12 patients. We present a 52-year-old female patient with a complaint of cervical mass, diagnosed with nasopharyngeal neuroendocrine carcinoma with pathological and radiological examinations. Panendoscopic upper respiratory tract examination of the patient showed a lesion suspicious of malignancy in the right Rosenmüller fossa. Biopsies performed from cervical lymphadenopathy and nasopharyngeal mass, revealed the diagnosis of poorly differentiated high-grade nasopharyngeal neuroendocrine carcinoma. The patient was referred to medical oncology department for chemotherapy and no recurrent lesion was found in the 1-year follow-up. Recognition of this rare tumor is important for disease management and patient prognosis. To draw attention to this rare clinical picture we would like to present our case in terms of diagnosis and treatment, together with a brief review of literature.
\end{abstract}

Keywords: neuroendocrine carcinoma, nasopharynx carcinoma, head and neck cancers

\footnotetext{
${ }^{1}$ Istanbul University - Cerrahpaşa, Cerrahpaşa Medical Faculty, Department of Otorhinolaryngology Head and Neck Surgery, Istanbul, Turkey

${ }^{2}$ Istanbul University - Cerrahpaşa, Cerrahpasa Medical Faculty, Department of Pathology, Istanbul, Turkey

* Corresponding author

E-mail: firattevetoglu@gmail.com; ORCID:0000-0002-1744-6995

Yetkin ZekiYllmaz: yetkin.yilmaz@iuc.edu.tr ORCID: 0000-0002-5734-9751,

HalukÇevik: haluk.cevik@iuc.edu.tr; ORCID: 0000-0002-5033-3255,

Cansu Yol: cansu.yol@iuc.edu.tr; ORCID:0000-0002-8151-2889,

Ayşegül Batıŏlu Karaaltın: batioglu@yahoo.com; ORCID: 0000-0002-8620-4375

Received: 20 November 2021 Revised: 30 November 2021 Accepted: 2 December 2021

DOI: 10.17932/EJOH.2020.022/ejoh_v02i2006
} 


\section{Introduction}

Neuroendocrine tumors are rare tumors that develop from neuroendocrine cells in the body. The most common sites are the lungs and the gastrointestinal tract (1). Neuroendocrine tumors are extremely rare in the head and neck region. The most common sites in the head and neck are larynx, sinonasal tract and parotid gland, respectively (2). Head and neck neuroendocrine carcinomas are usually seen in the 6th and 7th decades with prominent male predominance (3). Head and neck neuroendocrine carcinomas are classified according to WHO as such; poorly differentiated ( $>10$ mitosis/10 HPF), moderately differentiated (2-10 mitosis / 10 HPF with necrosis) and well differentiated (minimal nuclear atypia and $<2$ mitosis / 10 HPF). Poorly differentiated neuroendocrine carcinomas are further divided into two as large cell (LCNEC) and small cell neuroendocrine carcinoma (SCNEC). These malignant tumors have an aggressive course and a 5-year life expectancy of less than $20 \%$ (2).

Symptoms and signs in nasopharyngeal neuroendocrine carcinoma are similar to nasopharyngeal carcinoma; lymphadenopathy in the neck, especially in the posterior cervical triangle, nasal obstruction, epistaxis, serous otitis media due to Eustachian tube dysfunction. Among the etiological agents, smoking and human papilloma virus can be shown, which are also prevalent in squamous cell carcinoma of the oral cavity and oropharynx. While non-neuroendocrine tumors of the nasopharynx are largely associated with EBV, the role of EBV in the development of neuroendocrine carcinoma is not fully known. EBV is seen as a prognostic factor for nasopharyngeal malignancies. Treatment response and life expectancy are higher in EBV-positive nasopharyngeal carcinomas and lymphomas compared to EBV-negative malignancies (4).

In this case presentation, it was aimed to present this rare malignancy of head and neck region in the light of the literature and to provide additional information on the prognosis and follow-up, which were inadequately reported due to its rare occurrence.

\section{Case Presentation}

A 52-year-old female patient presented with the complaint of multiple swellings on the neck for 6 months. In physical examination, bilateral submandibular, level 2-3 lymphadenopathies were palpated. Endoscopic examination revealed a suspicious mass lesion in the right Rosenmüller fossa of nasopharynx. Ultrasonography (USG) examination revealed multiple lymphadenopathies in bilateral cervical lymphatic chain, with disappearance of the hilus formation, showing peripheral vascularity, with the largest being $38 \times 16 \mathrm{~mm}$. 
Contrast-enhanced nasopharyngeal magnetic resonance imaging (MRI) revealed thickening of the posterior wall of the nasopharynx and multiple lymphadenopathies at levels 1-2-3-4 of the neck (Figures 1A-1B). Intense FDG uptake (SUVmax: 24) in the right Rosenmüller fossa and a hypermetabolic nodular lesion with high suspicion for malignancy in the right parapharyngeal space (SUVmax: 16) was seen in positron emission tomography-computerized tomography (PET/CT) scan. In addition, conglomerated lymphadenopathies accompanied by intense FDG uptake (SUVmax: 28) were observed in bilateral cervical lymphatics. Moreover, multiple lymphadenopathies reaching $2 \mathrm{~cm}$ in size, with increased FDG intake in PET/CT in the right preauricular, right superficial parotid, bilateral supraclavicular lymph node stations and mediastinum, were observed.

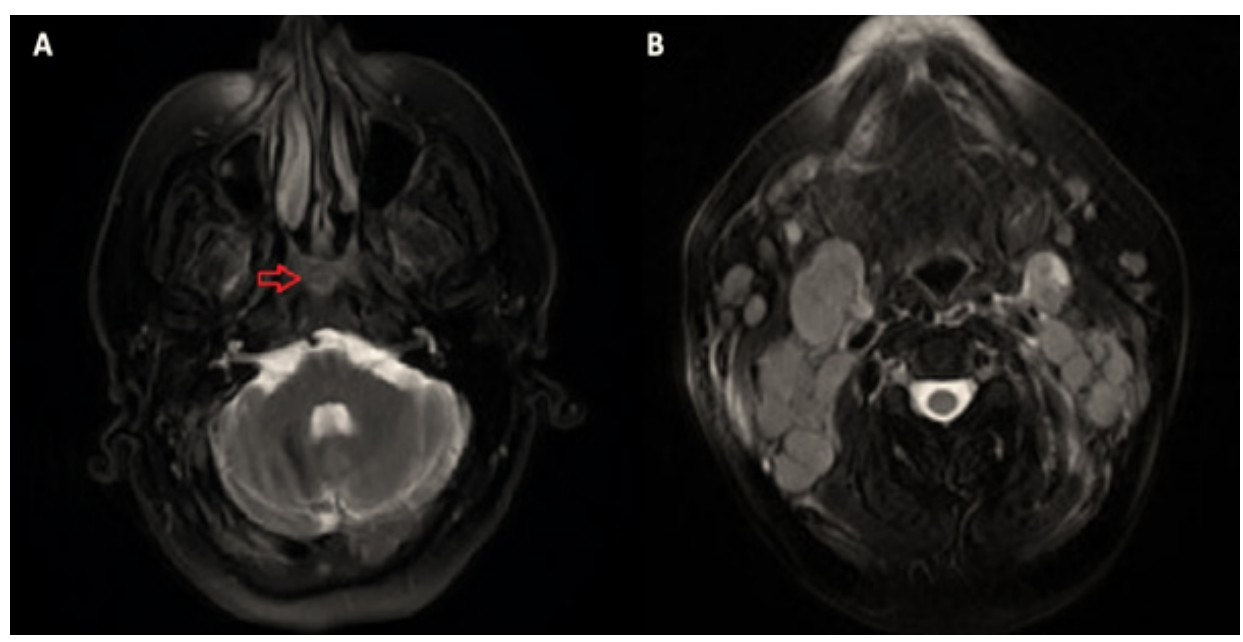

Figure 1. (A) Axial section of the nasopharyngeal MRI shows an asymmetrically thickness in the posterior wall of the nasopharynx at the right side. (B) Axial section of the cervical MRI shows multiple conglomerated lymphadenopathies in the bilateral cervical lymphatic chain.

Discohesive, atypical, pleomorphic cells which observed in fine needle aspiration cytology slides from cervical lymphadenopathy was interpreted as a metastasis of a malignant neoplasm. Histopathological examination of the incisional biopsy specimen from the nasopharynx revealed that the tumor cells formed a solid pattern with diffuse synaptophysin (Figure 2A) and cytokeratin-7 positivity (Figure 2B). EBER (Epstein-Barr virus-encoded small RNAs) and chromogranin was negative, while $\mathrm{p} 40$ showed focal nuclear positivity. In the light of these findings, according to WHO's classification, the patient was diagnosed with poorly differentiated (high-grade) carcinoma of the nasopharynx. 
The patient was scanned with 68Ga-DOTATATE PET/CT, which is a specific imaging method for neuroendocrine carcinoma. Since there was no other foci in the body expressing somatostatin receptor type 2, the lesion was interpreted as primary nasopharyngeal neuroendocrine carcinoma. Chemotherapy regimen which contains cisplatin + etoposide was applied to patient by medical oncology department. After a 1-year follow-up, PET/CT scan showed no pathological FDG uptake in nasopharynx and bilateral cervical lymphatics.
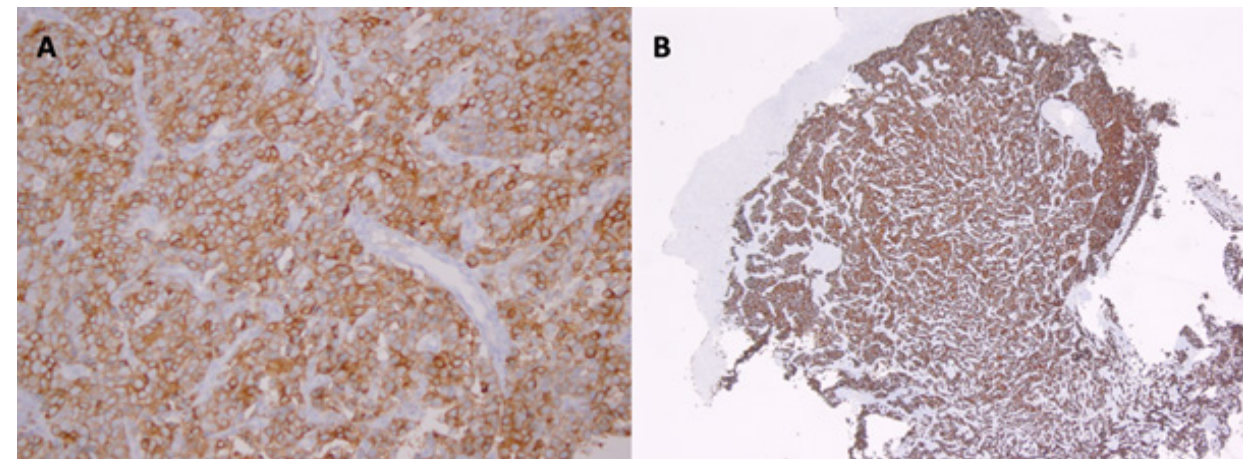

Figure 2. Light microscopic image. (A) Cytokeratin-7 positivity in tumor cells. (B) Synaptophysin positivity in tumor cells with immunohistochemical staining.

\section{Discussion}

Primary head and neck neuroendocrine carcinomas are extremely rare. Therefore in case of neuroendocrine carcinoma in a subsite of head and neck, primary versus metastasis differentiation should be made firstly $(5,6)$. Small cell carcinoma of the lung may be presented as extra-pulmonary neuroendocrine carcinoma focus due to its aggressive course. For this reason, while thorax CT had an important place in the approach to head and neck neuroendocrine carcinomas in the past, nowadays, whole body scanning should be performed with 68Ga-DOTATATE PET-CT, which is a neuroendocrine carcinoma specific imaging method.

The most common malignancy of the nasopharynx is non-keratinized undifferentiated carcinoma originating from the nasopharyngeal mucosal layer and is closely related to EBV. However nasopharyngeal neuroendocrine carcinoma is extremely rare. Among 12 cases reported to date, it was more common in males at a ratio of 10:2. Ten out of twelve cases reported to date have been reported as poorly differentiated. Three of these were reported as small cell neuroendocrine carcinoma, five as large cell neuroendocrine carcinoma and EBV was positive in three of the large cell neuroendocrine carcinoma cases $(3,7,8,9,10)$. In the other two cases, EBV serology is unknown. For this reason, it is thought that EBV may be responsible for large cell nasopharyngeal neuroendocrine carcinoma (3). 
It has been shown that the sensitivity of EBV-positive patients to chemotherapy and radiotherapy is higher than that of EBV-negative cases, so it has been estimated that EBV may be an important criterion in prognosis even if it did not play a role in etiology (3).

The treatment modality in head and neck neuroendocrine carcinomas depends on whether the tumor is resectable or unresectable or metastatic. Surgical treatment and adjuvant chemoradiotherapy is the treatment of choice for resectable tumors, while chemoradiotherapy alone is preferred for unresectable and metastatic tumors. Histopathologic characteristics of the tumor are also important factors for the treatment choice. While chemoradiotherapy is preferred primarily for small cell neuroendocrine carcinomas; surgery comes to the fore in non-small cell neuroendocrine carcinomas (11). In a 20 -year case follow-up study consisting of 16 cases; 11 of the patients had poorly-differentiated, 4 had moderatelydifferentiated, and 1 had well-differentiated neuroendocrine carcinoma in the head and neck region; with no statistically significant difference between moderately differentiated carcinoma and poorly differentiated carcinoma in terms of 2-year survival after treatment (\%37.5 vs. \%35.4) (12).

The follow-up of only 7 of 12 patients diagnosed with nasopharyngeal neuroendocrine carcinoma has been reported. In 6 cases of poorly differentiated nasopharyngeal neuroendocrine carcinoma with known follow-up, 2 patients were cured and 4 patients died despite treatment. In the case with well-differentiated nasopharyngeal neuroendocrine carcinoma, the patient was followed up with the disease for 7 years (3). In a case of small cell nasopharyngeal neuroendocrine carcinoma in 2007, a 43-year-old male patient died after 38 months of followup despite combined chemoradiotherapy (13). However, in another case report presented in 2011, a 41-year-old male patient diagnosed with small cell nasopharynx neuroendocrine carcinoma was said to be cured after 9 months of follow-up with combined chemoradiotherapy (14). Hence, the prognosis is expected to be poor especially in small cell nasopharyngeal neuroendocrine carcinoma due to poor differentiation, but there is insufficient data about the prognosis due to the low number of reported cases. Since the cases reported so far were mostly handled according to their histopathological characteristics, there is not enough information about follow-up and prognosis.

\section{Conclusion}

Nasopharyngeal neuroendocrine carcinoma is extremely rare. Most of the cases reported to date were poorly differentiated carcinomas and a significant proportion of them had a poor prognosis. Although nasopharyngeal neuroendocrine carcinomas are not among the common nasopharyngeal malignancies; it should be considered in the differential diagnosis of patients with complaints of hearing 
loss due to serous otitis media, nasal obstruction, epistaxis, neck mass especially in the posterior cervical triangle. Since it has poor prognosis, as far as we know, combined treatment with chemotherapy and concomitant radiotherapy should be initiated, as soon as possible.

\section{References}

1. Wolin EM. Advances in the Diagnosis and Management of Well-Differentiated and Intermediate-Differentiated Neuroendocrine Tumors of the Lung. Chest. 2017;151(5):1141-6.

2. El-Naggar AK, Chan JKC, Takata T, Grandis JR, Slootweg PJ. The fourth edition of the head and neck World Health Organization blue book: editors' perspectives. Hum. Pathol. 2017;66:10-2.

3. Cai Z, Lin M, Blanco AI, Liu J, Zhu H. Epstein-Barr Virus-Positive Large Cell Neuroendocrine Carcinoma of the Nasopharynx: Report of One Case and Review of the Literature. Head Neck Pathol 2019;13(3):313-7.

4. Ohiole Ozoya O, Sokol L, Dalia S. EBV-Related Malignancies, Outcomes and Novel Prevention Strategies. Infect Disord - Drug Targets 2016;16(1):4-21.

5. Bawa R, Wax MK. Small cell carcinoma of the tonsil. Otolaryngol - Head Neck Surg 1995;113(3):328-33.

6. Ibrahim NBN, Briggs JC, Corbishley CM. Extrapulmonary oat cell carcinoma. Cancer 1984;54(8):1645-61.

7. Chua MLK, Wee JTS, Hui EP, Chan ATC. Nasopharyngeal carcinoma. In: The Lancet. Elsevier Ltd; 2016. p. 1012-24.

8. Elloumi F, Fourati N, Siala W, et al. Tumeur neuroendocrine à grandes cellules du nasopharynx: À propos d'un cas. Cancer/Radiotherapie 2014;18(3):208-10.

9. Sturgis CD, Burkey BB, Momin S, Hoschar AP. High Grade (Large Cell) Neuroendocrine Carcinoma of the Nasopharynx: Novel Case Report with Touch Preparation Cytology and Positive EBV Encoded Early RNA. Case Rep Pathol 2015;2015:1-4.

10. Dumars C, Thebaud E, Joubert M, Renaudin K, Cariou-Patron G, Heymann MF. Large Cell Neuroendocrine Carcinoma of the Nasopharynx: A Pediatric Case. J Pediatr Hematol Oncol 2015;37(6):474-6.

11. Bacalao M, Beg MS, Cavuoti D, et al. Head and neck neuroendocrine tumors at a single institution over 15 years. Clin Case Reports 2019;7(12):2508-12. 
12. Meacham R, Matrka L, Ozer E, Ozer HG, Wakely P, Shah M. Neuroendocrine carcinoma of the head and neck: A 20-year case series. Ear, Nose Throat J 2012;91(3).

13. Lin IH, Hwang CF, Huang HY, Chien CY. Small cell carcinoma of the nasopharynx. Acta Otolaryngol 2007;127(2):206-8.

14. Lee LY, Chang KP, Hsu CL, Chen TC, Kuo TT. Small-cell neuroendocrine carcinoma of the nasopharynx: Report of a rare case lacking association with Epstein-Barr virus. Int J Surg Pathol 2011;19(2):199-202. 